\title{
EVALUACIÓN DE LA APLICACIÓN DE LAS GUÍAS DE HIPERTENSIÓN Y DIABETES EN UN PROGRAMA DE CRÓNICOS
}

\author{
Juan C. Ávilai, Adriana Bareñoㅇ, Jairo Castro3, Catherine Rojast. \\ ${ }^{1}$ MSc., Profesor Asociado, Facultad de Medicina, Universidad Militar Nueva Granada. \\ ${ }^{2}$ EsP., Directora de auditoría médica regional Bogotá - Sanitas \\ ${ }^{3} \mathrm{MD}$, Médico Joven Investigador, Facultad de Medicina Universidad Militar Nueva Granada \\ ${ }^{4}$ Estudiante, Facultad de Medicina, Universidad Militar Nueva Granada. \\ Correspondencia: juan.avila@unimilitar.edu.co
}

Recibido: Febrero 4 de 2014 Aceptado: Julio 17 de 2014

\begin{abstract}
Resumen
Introducción: La Hipertensión arterial (HTA) y la Diabetes Mellitus (DM) hacen parte de las enfermedades crónicas no trasmisibles (ECNT) y ocupan las primeras causas de morbimortalidad a nivel mundial y nacional. Por tal razón es importante el control de estas enfermedades para prevenir la progresión de las coomorbilidades, con un impacto directo en la calidad de vida y en los costos de atención del paciente, por esto es importante evaluar la correcta aplicación de las guías de HTA y DM en los programas de prevención y control de las ECNT.

Metodología: Se realizó un estudio descriptivo transversal por medio de un muestreo aleatorio estratificado. Para evaluar el control de los pacientes según la aplicación de las guías de Hipertensión y Diabetes en una población de usuarios inscritos en los programas de promoción y prevención de una Entidad Adaptada al Sistema, en la ciudad de Bogotá.

Resultados: Se obtuvo una población de 425 pacientes hipertensos y de 279 diabéticos con un nivel de confianza de $95 \%$ y una precisión del $80 \%$. Se encontró en el programa de hipertensos un cumplimiento de la guía del $67,8 \%$ y un control de la enfermedad del $60 \%$. De los pacientes diabéticos evaluados se encontró un cumplimiento de la guía del $62,8 \%$ y un control de la patología menor al $1 \%$ y un aceptable control del $51 \%$.

Conclusión: Con el porcentaje de cumplimiento encontrado en la aplicación de las guías se está logrando un control en el programa de hipertensión de acuerdo a lo esperado, sin embargo en el programa de pacientes diabéticos se encuentra un bajo control. Por tal razón es imperativo mejorar la aplicación de las guías de manejo de estas patologías y así impactar en el control de la enfermedad y la calidad de vida de los pacientes.

Palabras clave: Hipertensión, diabetes mellitus, insuficiencia renal, tasa de filtración glomerular.
\end{abstract}




\title{
HYPERTENSION AND DIABETES GUIDELINES EVALUATION AND APPLICATION IN A CHRONIC DISEASE PROGRAM
}

\begin{abstract}
Introduction: The hypertension and Mellitus diabetes are two of the highly risky diseases that are not contagious and are the first causes of mortality in an international and national level. For this reason is important to control these diseases to prevent the progression of the illnesses, with a direct impact in the life style and in the costs of the services for the patients, because of this is important to evaluate the right use of the HTA and DM guides in the prevention and control programs of the ECNT.

Methodology: A transversal descriptive study was run by a random stratified sampling. To evaluate the control of the patients according to the application of the HTA and DM guides in a group of users of the promotion and prevention programs of a medical services institution in Bogota.

Results: A group of 425 Hypertension patients and 279 diabetic patients was obtain; with a trusting level of $95 \%$ and a precision level of $80 \%$. In the hypertension program was found a fulfillment of the guide of $67.75 \%$ and a $60 \%$ of the disease control. About the diabetic patients that were evaluated was found a fulfillment of the guide of $62.75 \%$, less of $1 \%$ pathology control and an acceptable control of $51 \%$.

Conclusions: With the application guide percentage is being achieved the control in the hypertension program according to what was expected, but in the program of diabetic patients was found an underachieve. For this reason a better application of the guides is needed for the control of these diseases and because of this an impact in the illness control and life style of the patients.
\end{abstract}

Key words: Hypertension, diabetes mellitus, renal insufficiency, glomerular filtration rate.

\section{AVALIAÇÃO DA IMPLEMENTAÇÃO DAS GUIAS DE HIPERTENSÃO E DIABETES EM UM PROGRAMA DE PACIENTES CRÔNICOS}

\begin{abstract}
Resumo
Introdução: A hipertensão arterial (HTA) e a Diabetes mellitus (DM) fazem parte das doenças crônicas não transmissíveis (DCNT), sendo causas primarias de morbimortalidade no nível mundial e nacional. Além disso, é importante o controle destas doenças com o fim de prevenir a progressão das comorbilidades, com um impacto direito na qualidade de vida e nos custos da atenção dos doentes. Pelo anterior, é importante avaliar a correta implementação das Guias de HTA e DM nos programas de prevenção e controle das DCNT.

Metodologia: Foi feito um estudo descritivo, transversal, aleatório estratificado, com o fim de avaliar o controle dos pacientes crônicos segundo a implementação das Guias de HTA e DM em uma população de usuários inscritos nos programas de promoção e prevenção de uma entidade adaptada ao sistema, na cidade de Bogotá.
\end{abstract}


Resultados: Obteve-se uma população de 425 pacientes com HTA e de 279 com DM, com um nível de confiança de $95 \%$ e uma precisão de 80\%. A implementação da Guia foi de 67,8\% nos pacientes com HTA, com $60 \%$ de controle da doença. Nos pacientes com DM, a implementação da guia foi de $62,8 \%$, controle da doença menor de $1 \%$ e controle aceitável de $51 \%$.

Conclusão: Segundo a porcentagem obtida da implementação das Guias, está-se logrando um controle no programa de HTA, embora no programa de pacientes com DM existe um baixo controle. É por isso que é importante melhorar a implementação das guias destas doenças, com o fim de impactar no controle da doença mesma e a qualidade de vida dos pacientes.

Palavras-chave: Hipertensão, diabetes mellitus, insuficiência renal, taxa de filtração glomerular.

\section{Introducción}

Las Enfermedades Crónicas no Trasmisibles (ECNT) son las responsables del $60 \%$ de las muertes a nivel mundial y representan el doble del número de muertes causadas por las enfermedades infecciosas, patologías maternas y perinatales, y las debidas a carencias nutricionales. Dentro del grupo de ECNT, la Hipertensión Arterial y la Diabetes Mellitus ocupan las primeras causas de morbimortalidad en nuestro país y actúan como predictores para el desarrollo de la Insuficiencia Renal Crónica (IRC) (1).

La IRC es considerada una patología de alto costo, la cual puede ser prevenible con una adecuada implementación de programas de promoción y prevención. Teniendo como referencia en Colombia las guías de atención de hipertensión arterial y diabetes mellitus, desarrolladas por el Ministerio de Protección Social mediante la resolución 412 del año $2010(2,3)$.

El no cumplimiento de las guías implica una progresión más rápida de la enfermedad con impacto directo en la calidad de vida y en los costos de atención del paciente, por ello se destaca la importancia de evaluar la correcta aplicación de las guías de HTA y DM $(2,3)$ en los programas de prevención y control de las ECNT en una Entidad Adaptada al Sistema General de Seguridad Social en Salud, en la ciudad de Bogotá. En este estudio y a partir de un muestreo aleatorio estratificado de la población adscrita a los programas de promoción y prevención de los usuarios de una institución prestadora de servicios de salud adscrita a una Entidad Adaptada al Sistema, en la ciudad de Bogotá, por medio de la revisión directa de la historia clínica se evaluó de manera objetiva y cuantitativa la efectividad de la aplicación de las guías y el nivel de control de los pacientes en sus patologías de base.

\section{Metodología}

Como objetivo se propuso evaluar el control de los pacientes con Hipertensión Arterial y Diabetes Mellitus tipo 2 de acuerdo a las guías de los programas de enfermedad crónica en los usuarios inscritos en el programa de promoción de la salud y prevención de la enfermedad de los usuarios de una institución prestadora de servicios de salud adscrita a una Entidad Adaptada al Sistema, en la ciudad de Bogotá.

Se realizó un estudio descriptivo transversal para la evaluación del control de los pacientes según las guías de HTA y DM 2 en la población de usuarios inscritos en los programas de promoción y prevención.

\section{Cálculo de la muestra}

Se contó con un universo de 332 historias clínicas de los pacientes del programa de Diabetes Mellitus tipo 2 y de 617 pacientes del programa de Hipertensión.

Usando el software EPIDAT se calcularon los tamaños de las muestras para tener un nivel de confianza de 95\% y una precisión del $80 \%$, teniendo en cuenta una proporción esperada de pacientes controlados del $20 \%$ para los Diabéticos según el análisis de la cuenta de alto costo para Colombia (4) y del 62\% para los Hipertensos de acuerdo al informe nacional de calidad de la atención en Salud del 2009 que mostro un control entre el 62-72\% según informes de las instituciones prestadoras de salud (5).

Se obtuvo una muestra de 274 pacientes del programa de diabetes y de 455 pacientes del programa de Hipertensión.

\section{Criterios de inclusión}

- Paciente inscrito en el programa de Enfermedad Crónica (HTA y/o DM) 


\section{Criterios de exclusión}

- Paciente con menos de 1 año de antigüedad en el programa.

- Pacientes que se encuentran en el programa de crónicos con diagnósticos diferentes a los del objeto del estudio.

Sobre la historia clínica del paciente y tomando los registros del último año (a partir de la fecha de la última consulta en el programa) se aplica un instrumento previamente diseñado y validado que contempla aspectos como: datos socio-demográficos, signos vitales, medidas antropométricas, examen físico completo, laboratorios, tratamiento, adherencia al tratamiento, frecuencia de controles y valoraciones por especialistas. Los datos obtenidos de las historias revisadas fueron analizados en el programa STATA.

Teniendo en cuenta que la tasa de filtración glomerular debe estimarse con ecuaciones de predicción que contengan las variables: creatinina sérica, edad, sexo, raza y con los datos tomados del análisis de la historia clínica, se realiza el cálculo de la Tasa de filtración glomerular por medio de la ecuación abreviada del MDRD.

$$
T F G=186 \times\left(\text { creatininaserica }^{(-1.154)}\right) \times\left(\text { edad }^{(-0.203)}\right)
$$

Para mujeres se multiplica por 0.742 y para personas de raza negra se multiplica por 1.21 .

\section{Aspectos éticos}

La presente investigación se clasifica como una investigación sin riesgo, ya que la información recolectada se basa en registros y no se realiza ninguna intervención o modificación de las variables del individuo, por lo tanto no requiere Comité de Ética ni consentimiento informado.

\section{Resultados}

\section{Programa de hipertensión}

Se presentó del total de la muestra una pérdida del 7\% de historias clínicas, algunas de ellas se encontraban en el archivo muerto y otras en archivos de otras ciudades, finalmente se contó con un total de historias clínicas como se muestra en la tabla 1.

\section{Frecuencia controles}

Se encontró un cumplimiento en la frecuencia de los controles como se muestra en la tabla 2 de acuerdo a lo esti-
Tabla 1. Historias clínicas evaluadas y distribución por género grupo de pacientes hipertensos.

\begin{tabular}{|l|c|c|}
\hline \multicolumn{1}{|c|}{ Genero } & $\mathbf{N}^{\circ}$ de pacientes & Edad promedio \\
\hline Femenino & 271 & 70 \\
\hline Masculino & 154 & 72 \\
\hline Total & 425 & 71 \\
\hline
\end{tabular}

pulado en la guía. De los pacientes que no cumplieron con la asistencia sugerida al programa el $63 \%$ acudió en menor frecuencia y el $37 \%$ con mayor frecuencia a lo sugerido.

\section{Historia clínica}

De los aspectos a evaluar contemplados en la guía y consignados con mayor frecuencia en la historia clínica fueron los antecedentes, tratamiento dado, signos vitales e Índice de Masa corporal (IMC). Los menos evaluados fueron los relacionados con la búsqueda de daño a órgano blanco (fondo de ojo, pulsos periféricos y valoración neurológica), como se muestra en la tabla 2.

Tabla 2. Evaluación aspectos de la historia clínica.

\begin{tabular}{|l|c|c|c|c|}
\hline Aspecto evaluado & Cumple & \% & $\begin{array}{c}\text { No } \\
\text { cumple }\end{array}$ & \% \\
\hline $\begin{array}{l}\text { Sintomas tiempo de } \\
\text { evolución }\end{array}$ & 239 & 56 & 186 & 44 \\
\hline Antecedentes & 398 & 94 & 27 & 6 \\
\hline $\begin{array}{l}\text { Evaluacion factores de } \\
\text { riesgo cardiovascular }\end{array}$ & 289 & 68 & 136 & 32 \\
\hline $\begin{array}{l}\text { Tratamientos previos y } \\
\text { efectos secundarios }\end{array}$ & 403 & 95 & 22 & 5 \\
\hline Fondo de ojo (anual) & 142 & 33 & 283 & 67 \\
\hline Pulsos (anual) & 41 & 10 & 384 & 90 \\
\hline Valoración neurologica & 72 & 17 & 535 & 83 \\
\hline $\begin{array}{l}\text { Evaluacion de daño a } \\
\text { organo blanco (anual) }\end{array}$ & 367 & 86 & 58 & 14 \\
\hline Singnos vitales & 416 & 98 & 9 & 2 \\
\hline $\begin{array}{l}\text { Peso -indice de masa } \\
\text { corporal }\end{array}$ & 385 & 91 & 40 & 9 \\
\hline Examen fisico & 340 & 80 & 85 & 20 \\
\hline Promedio & $66 \%$ & & $34 \%$ & \\
\hline
\end{tabular}

Como hallazgo se observó que los médicos familiares y médicos generales que pertenecen al programa de crónicos son quienes elaboran las historias clínicas más completas en comparación con los médicos que no pertenecen al programa. Además las historias realizadas por especialista o profesionales con segunda especialidad fueron las que más fallas presentaban en el registro. Uno de los factores limitantes de la revisión fue la ilegibilidad de las historias 
clínicas escritas a mano, impidiendo una compresión adecuada de la información consignada.

\section{Ayudas diagnosticas}

El cumplimiento en solicitud de ayudas diagnosticas es del 61\%; los paraclínicos más solicitados fueron el perfil lipídico y la glicemia. Además se encontró un adecuado reporte de creatinina en las historias clínicas, pero no se observó la tasa de filtración glomerular o la estratificación de los pacientes. Dentro de los paraclínicos menos solicitados están el parcial de orina (parámetro de detección de proteinuria, hematuria y glucosuria). Otro de los exámenes de seguimiento menos solicitado fue el electrocardiograma, tabla 3.

Tabla 3. Cumplimiento del control con ayudas diagnósticas.

\begin{tabular}{|l|c|c|c|c|}
\hline Aspecto evaluado & Cumple & $\mathbf{\%}$ & $\begin{array}{c}\text { No } \\
\text { cumple }\end{array}$ & $\mathbf{\%}$ \\
\hline Perfil lipídico & 343 & 81 & 82 & 19 \\
\hline Uroanálisis & 296 & 70 & 129 & 30 \\
\hline Creatinina & 313 & 74 & 112 & 26 \\
\hline Glicemia & 321 & 76 & 104 & 24 \\
\hline Electrocardiograma & 136 & 32 & 289 & 68 \\
\hline Potasio & 276 & 65 & 149 & 35 \\
\hline Otros & 122 & 29 & 303 & 71 \\
\hline Promedio & $61 \%$ & & $39 \%$ & \\
\hline
\end{tabular}

\section{Tratamiento}

$\mathrm{Al}$ evaluar las indicaciones de tratamiento para cada paciente según lo sugerido en las guías de manejo, se pudo observar que el personal de la salud a cargo del programa tiene claro el esquema de manejo para cada individuo, mostrando un $99 \%$ de cumplimiento para este factor. En lo concerniente a las recomendaciones de su enfermedad de base, estilos de vida y tratamientos se observó que en el 98\% fueron mencionadas. Adicionalmente como hallazgo positivo se encontró en algunas historias clínicas reportes de la Automedicación de la Presión Arterial (MAPA) para el monitoreo de las cifras tensionales de los pacientes.

La frecuencia de remisiones (medicina interna, nefrología, nutrición y optometría) según la guía o necesidad del paciente mostro un cumplimiento del $54 \%$.

\section{Control de los pacientes}

De la totalidad de las historias clínicas revisadas y de acuerdo a los parámetros establecidos en las guías de manejo se observó un control del $79 \%$ de los pacientes pertenecientes al programa de hipertensos, con un porcentaje promedio de cumplimiento de la guía del $60 \%$.

\section{Análisis diabetes}

Frecuencia controles

Los pacientes con diagnóstico de diabetes mellitus deben tener un seguimiento estrecho debido a los cambios oscilantes y frecuentes de las cifras de glicemia, para disminuir el daño de órgano blanco con las hiperglicemias. Se encuentra un buen porcentaje de cumplimiento a los controles permitiendo un adecuado seguimiento de los pacientes por parte del programa, como se muestra en la tabla 4, pero aún se evidencia que un $23 \%$ de los pacientes no asisten con la frecuencia adecuada, por lo que esta población debe ser captada por el programa para hacer un óptimo seguimiento de su patología, disminuyendo de esta manera la progresión de la enfermedad renal. De los pacientes que no cumplieron con el parámetro los controles, el $84 \%$ fue por asistir en menor frecuencia.

Tabla 4. Cumplimiento en la frecuencia de los controles.

\begin{tabular}{|l|c|c|}
\hline \multicolumn{3}{|c|}{ FRECUENCIA DE CONTROLES PACIENTES CON DM 2} \\
\hline & Pacientes & Procentaje (\%) \\
\hline Cumple & 216 & 77 \\
\hline No cumple & 63 & 23 \\
\hline Total & 279 & 100 \\
\hline
\end{tabular}

\section{Historia clínica}

El cumplimiento general en calidad de historia clínica es del 69\%, cifra mayor en comparación al programa de hipertensión. Pero de los aspectos que no están siendo evaluados se encuentran los síntomas de evolución de la enfermedad, el examen de pies y la valoración del daño micro-vascular y neurológico (pulsos, sensibilidad y reflejos), tabla 5 .

Tabla 5. Evaluación aspectos de la historia clínica pacientes con Diabetes Mellitus.

\begin{tabular}{|l|c|c|c|c|}
\hline \multicolumn{1}{|c|}{ Aspecto evaluado } & Cumple & $\mathbf{\%}$ & $\begin{array}{c}\text { No } \\
\text { cumple }\end{array}$ & $\mathbf{\%}$ \\
\hline Sintomas & 126 & 45 & 153 & 55 \\
\hline Adherencia al tratamiento & 243 & 87 & 36 & 13 \\
\hline Signos vitales & 274 & 98 & 5 & 2 \\
\hline Peso-imc & 250 & 90 & 29 & 10 \\
\hline Evaluación pies & 112 & 40 & 167 & 60 \\
\hline Pulsos-sensibilidad y reflejos & 39 & 14 & 240 & 86 \\
\hline Actualización historia clínica & 218 & 78 & 61 & 22 \\
\hline Adecuado registro & 271 & 97 & 8 & 3 \\
\hline Promedio & 69 & & 31 & \\
\hline
\end{tabular}


También se observó la adecuada detección de pacientes con sobrepeso y obesidad pero no se evidencia del seguimiento ni control para que el paciente llegue a un estado nutricional adecuado.

\section{Valoraciones adicionales}

El cumplimiento general en valoraciones adicionales es del $19 \%$, se esperaría que todos los pacientes tengan una valoración por cada una de estas áreas, de este modo se podría tener una detección temprana del daño de retina, el control del estado nutricional y hábitos alimenticios de los pacientes. Muchas de las valoraciones encontradas de optometría y oftalmología fueron para manejo de patologías oculares más no de tamizaje. En ninguna de las historias revisadas se encontró valoración por odontología, por lo que se puede inferir que se lleva una historia clínica diferente para odontología, ver tabla 6 .

Tabla 6. Solicitud de otras valoraciones

\begin{tabular}{|l|r|r|c|c|}
\hline Aspecto evaluado & Cumple & $\mathbf{\%}$ & $\begin{array}{c}\text { No } \\
\text { cumple }\end{array}$ & $\mathbf{\%}$ \\
\hline Oftalmología & 116 & 42 & 163 & 58 \\
\hline Nutrición & 45 & 16 & 234 & 84 \\
\hline Odontología & 1 & 0 & 278 & 100 \\
\hline Promedio & 19 & & 81 & \\
\hline
\end{tabular}

Control ayudas diagnósticas

El cumplimiento general de ayudas diagnosticas para seguimiento y control fue del 35\%para estos pacientes. De estos laboratorios la hemoglobina glicosilada A1c es uno de los parámetros que nos permite mejor control del paciente diabético, se encontró que el 39\% de los pacientes del programa estuvieron controlados y el $13 \%$ en aceptable control. No se está tomando glicemia postprandial, examen relevante para el ajuste de los esquemas de manejo dietario y farmacológico de estos pacientes, Tabla 9.

Llama la atención que cerca de la mitad de los pacientes pertenecientes al programa de diabetes presenta un IMC elevado, pero no se evidenciaron medidas efectivas para su control, limitando así el manejo de su patología de base $e$ incrementando el riesgo de enfermedad cardiovascular, Tabla 9.

Los laboratorios para detección temprana de insuficiencia renal como el parcial de orina y la microalbuminuria no se están solicitando de acuerdo a lo sugerido, Tabla 7.

\section{Tratamiento}

El cumplimiento general en cuanto a la indicación de tratamiento a los pacientes fue del $78 \%$, y las recomendaciones dadas a los pacientes acerca del tratamiento, alimentación y estilos de vida fue del $94 \%$.

\section{Control de los pacientes}

Se encontró que solo uno de los pacientes del programa está controlado con un cumplimiento en la historia clínica del $71 \%$, cuando se esperaba que el $20 \%$ de la población estuviera en control de acuerdo a lo reportado en los análisis de la cuenta de alto costo (4), tabla 8.

\section{Discusión}

Las personas con HTA tienen 2-4 veces más riesgo de presentar enfermedad coronaria (EC) y 10-20 veces mayor riesgo de presentar un accidente cerebrovascular (ACV)

Tabla 7. Cumplimiento del control con ayudas diagnósticas.

\begin{tabular}{|c|c|c|c|c|c|c|}
\hline Aspecto evaluado & Controlado & $\%$ & $\begin{array}{c}\text { Aceptable } \\
\text { control }\end{array}$ & $\%$ & $\begin{array}{c}\text { No } \\
\text { controlado } \\
\end{array}$ & $\%$ \\
\hline Tensión arterial & 238 & 85 & 20 & 7 & 21 & 8 \\
\hline Índice de masa corporal & 69 & 25 & 43 & 15 & 128 & 46 \\
\hline Glicemia & 75 & 27 & 48 & 17 & 118 & 42 \\
\hline Glicemia 2 horas postpandrial & 38 & 14 & 20 & 7 & 74 & 27 \\
\hline Hemoglobina glicosilada (hb1c) & 110 & 39 & 37 & 13 & 75 & 27 \\
\hline Colesterol ldl & 104 & 37 & 31 & 11 & 64 & 23 \\
\hline Colesterol hdl & 134 & 48 & 35 & 13 & 41 & 15 \\
\hline Triglicéridos & 110 & 39 & 29 & 10 & 70 & 25 \\
\hline Creatinina & 151 & 54 & 1 & 0 & 27 & 10 \\
\hline Microalbuminuria & 19 & 7 & 1 & 0 & 4 & 1 \\
\hline Parcial de orina & 3 & 1 & 115 & 41 & 11 & 4 \\
\hline Promedio & 34 & & 12 & & 21 & \\
\hline
\end{tabular}


Tabla 8. Grado de control y cumplimiento de la guía de diabetes.

\begin{tabular}{|l|c|c|c|}
\hline $\begin{array}{c}\text { Grado de } \\
\text { control }\end{array}$ & Pacientes & $\begin{array}{c}\text { Porcentaje } \\
\text { de } \\
\text { pacientes }\end{array}$ & $\begin{array}{c}\text { Porcentaje } \\
\text { de cumpli- } \\
\text { miento de } \\
\text { guía }\end{array}$ \\
\hline Aceptable control & 142 & 51 & 64 \\
\hline No controlado & 136 & 49 & 58 \\
\hline Controlado & 1 & 0 & 71 \\
\hline Total & 279 & 100 & 64 \\
\hline
\end{tabular}

por aumento de la presión arterial diastólica, pero se ha demostrado que el adecuado control de la HTA disminuye en un $20-25 \%$ el riesgo de enfermedad coronaria y del 30-50\% el riesgo de accidente cerebrovascular, por tal razón controlar la tensión arterial es disminuir la prevalencia de EC, ACV, mejorar la calidad y expectativa de vida de la población (5).

En este estudio se encontró un control del 79\% de los pacientes que se encuentran inscritos en el programa de hipertensos cifra que está por encima del Informe Nacional de Calidad de la Atención en Salud del 2009 que mostró un control de $62-72 \%$ según informes de las instituciones prestadoras de salud. Reportes del estudio NHANES III (Third National Health and Nutrition Examination Survey) afirman que aproximadamente el $32 \%$ de las personas hipertensas no conocen su condición y no reciben tratamiento, el 15\% conocen su enfermedad pero no reciben tratamiento, el $26 \%$ son tratados pero no llegan a controlar sus valores de tensión arterial y solo el $27 \%$ controlan sus cifras tensiónales $(1,2)$. En un estudio realizado por Ferrera N. en Panamá en el año de 2010, con 79 pacientes hipertensos, encontró que el 39\% presentó cifras de presión arterial (PA) en rango normal o limítrofe y el 37\% se encontraba en estadio I (7), cifras inferiores a lo obtenido en el presente estudio.

Se observó un cumplimiento general del $60 \%$ de la guía lo cual se traduce en un $79 \%$ del control de los pacientes, cifras que comparadas con otros estudios a nivel mundial son alentadoras, sin embargo se hace necesario impactar en los aspectos de menor fortaleza con el fin de lograr un mayor control de los pacientes impactando en la calidad de vida de nuestra población.

En un estudio realizado en Manizales en el periodo de 2004-2007 acerca de la calidad en historias clínicas de pacientes hipertensos, se encontró un cumplimiento a los controles del 39,6\%, con una inasistencia del 60,4\% (8). En el presente estudio se encontró un mayor cumplimiento de los pacientes a los controles con un $62 \%$.
Uno de los hallazgos relevantes fue la ilegibilidad de la historia clínica y en algunos casos no se registraron completamente los datos, situación que dependió del profesional de la salud que la realizo; limitando de esta manera la interpretación de la historia clínica al personal que requiere consultarla posteriormente. En canto a este aspecto se hace necesario tener en cuenta la resolución 1995 de 1999 por la cual se establecen las normas para el manejo de la historia clínica en Colombia.

Un 38\% de los pacientes no asistió a los controles de acuerdo a lo estipulado en la guía, de estos el $63 \%$ con una menor frecuencia y el $37 \%$ con una mayor frecuencia. Aspecto importante para lograr un impacto positivo sobre la morbilidad de los pacientes por lo cual se hace necesario plantear estrategias que permitan mitigar dicho hallazgo.

Un estudio hecho por Prado H. Ciudad Bolivar- Venezuela en Agosto 2010 busco evaluar el conocimiento acerca de su enfermedad a los pacientes (síntomas, cifras tensiónales normales, estilos de vida saludables, coomorbilidades, complicaciones e importancia del tratamiento) pertenecientes a un programa de hipertensos el cual mostro que el 57,1\% no tenía ningún conocimiento, el $8 \%$ poco conocimiento y apenas el $1 \%$ tenía el suficiente conocimiento respecto a su patología (9). Esto nos permite predecir que los pacientes que no saben de su enfermedad van a fallar en aspectos como la frecuencia de los controles, adherencia al tratamiento, control de los factores de riesgo y de las cifras tensiónales.

En lo concerniente a los laboratorios el 61\% estuvo acorde a lo estipulado en la guía y de estos los menos solicitados fueron el electrocardiograma (ECG), potasio y el parcial de orina, y es importante tener en cuenta que el inadecuado apoyo en los laboratorios impide la detección precoz del daño a órgano blanco causado por la enfermedad. Papazafiropoulou A. en 2011 evaluó la prevalencia de daño de órgano blanco en 611 pacientes hipertensos en atención primaria, encontrando una mayor prevalencia en hombres que en mujeres, además hipertrofia ventricular izquierda de 33\% en la población, engrosamiento de la capa intima carotidea en el 21,8\% de los pacientes, elevación de la creatinina plasmática en el $11 \%$ de los pacientes y microalbuminuria de 14,6\% de los pacientes. Estudios realizados en EE.UU., África y Europa también han mostrado una alta prevalencia, además de asociarse con una mayor mortalidad cardiovascular. Por tal razón se debe solicitar exámenes como el ECG y el parcial de orina de acuerdo a lo establecido en la guía (10).

Una de las fallas encontradas y de vital importancia es la evaluación de órgano blanco en el examen físico; en el 
presente estudio se observó un incumplimiento del 90\% en la evaluación de pulsos periféricos, del $83 \%$ en la valoración neurológica y del $67 \%$ en el fondo de ojo. Esto se correlaciona con una inadecuada tamización del daño micro-vascular de los órganos blanco en la población. Otros estudios demuestran una prevalencia del 29,6\% para enfermedad vascular en estas poblaciones $(8,11)$, por tal motivo se debe hacer énfasis en el control de estos aspectos.

Se encontró que el 99\% de la población de hipertensos cumple con el consumo de la medicación dada y el 98\% con las recomendaciones, según lo reportado en las historias clínicas. Pero es importante conocer los conceptos de los pacientes acerca de su enfermedad, de los medicamentos que toman, las recomendaciones dadas y su aplicación, además de las creencias propias y las infundadas por otras personas.

La diabetes mellitus es una enfermedad de alta prevalencia, con elevadas tasas de morbilidad y mortalidad. Su aparición, así como el desarrollo y la gravedad de sus complicaciones están relacionados con el diagnóstico oportuno y al adecuado control de los niveles de glucosa. Los estudios han documentado que tanto los programas de atención como la adherencia de los pacientes al tratamiento son determinantes en el control del paciente (4).

En el 2011 las federaciones internacionales de diabetes "lanzan un plan mundial contra la diabetes para el periodo de 2011-2021", con el objetivo de lograr mejorar la salud y los resultados relacionados con la calidad de vida para de estos pacientes, reducir los costos personales y sociales, y el impacto negativo sobre el desarrollo sostenible humano y económico. Fue diseñada para estar al alcance y la capacidad de todos los países. En ella se definen los componentes clave de una atención diabética eficaz: 1 . Tratamiento y monitorización clínica para lograr control glucémico y metabólico. 2. Educación y apoyo para el autocontrol. 3. Prevención y control de complicaciones (12).

En la frecuencia a los controles del programa de pacientes con diabetes se encontró un mayor cumplimiento en comparación al programa de hipertensos, con un porcentaje del 77\%, explicado probablemente porque la patología requiere un seguimiento más estrecho. En este estudio no se evaluó ni el nivel educativo, ni el conocimiento de la enfermedad en la población. Dado que es un determinante importante en la frecuencia de controles, adherencia al tratamiento y por ende el control de la enfermedad. Estudios han mostrado que los programas de educación a los pacientes para incrementar el grado de conocimiento de la enfermedad y su manejo han logrado un control de la dia- betes entre el 35,7-48,7\% de los pacientes pertenecientes a un programa de crónicos (13).

Factores de seguimiento de pacientes diabéticos como síntomas de la enfermedad y evaluación de órgano blanco tiene bajas cifras de cumplimiento en este programa, con apenas un cumplimiento del $45 \%$ de evaluación de síntomas de la enfermedad, 40\%, evaluación de pies y del $14 \%$ en la evaluación de pulsos, sensibilidad y reflejos. En un estudio realizado en Europa con 1819 pacientes en un periodo de 7 años, encontró una incidencia de neuropatía del 23,5\%. Además se encontró que la diabetes más los factores de riesgo cardiovascular incrementa la prevalencia de neuropatía (14). Otros estudios han mostrado incidencias de retinopatía diabética entre un 7,3 y $10,4 \%$ en pacientes diabéticos con una terapia intensiva y terapia estándar respectivamente, con una hemoglobina glicosilada blanco menor de 6\% para la terapia intensiva y menos de 7,9\% para la terapia estándar (15). De los pacientes evaluados en el programa solo uno se encuentra en adecuado control y el resto está en aceptable control o no controlado lo que aumenta la prevalencia de retinopatía en esta población, además se encuentra una baja valoración del fondo de ojo de tamizaje; solo el $42 \%$ lo cumple, por tal razón de no realizar un control estrecho y a tiempo del daño a órgano blanco se disminuirá la calidad de vida de los pacientes y aumentaran los costos de manejo para el paciente y el sistema de salud.

Los pacientes del programa de diabetes deben tener un adecuado control de los hábitos alimenticios, del perfil lipídico y de su peso corporal, por este motivo es importante un seguimiento de acuerdo a la guía por parte de nutrición y el médico tratante. Se observó que el 16\% de estos pacientes tuvieron una valoración por nutrición, esta situación favorece el desarrollo de cuadros como el síndrome metabólico con desenlaces como infartos cerebrales, cardiacos o el daño a órgano blanco.

Una de las consecuencias de la DM es el daño renal y por ende es importante hacer el tamizaje de acuerdo a la guía con el parcial de orina y microalbuminuria. Se encontró en este programa que en un 54\% y $91 \%$ de los usuarios respectivamente, no se solicitó el laboratorio. En un estudio realizado por Sánchez D. en México publicado en 2012, encontró en un programa de pacientes con diagnóstico de DM e HTA que el 32\% de esa población presentó microalbuminuria, proporción semejante a la reportada por otros autores. Se aprecia además que más del 55\% de la población tiene una relación albúmina/creatinina normal; sin embargo, el resto de la población ya presenta cifras alteradas (16). 
Según la población estudiada el control de la diabetes está por debajo de lo reportado en la Cuenta de Alto Costo, en donde se menciona un control del $20 \%$. Otros estudios muestran un control casi del 50\% tanto de en glicemia basal, hemoglobina glicosilada, presión arterial y perfil lipídico (13). Sánchez P. en 2010 encontró en un programa de diabéticos tipos 2 un control del 9,42\% en hombres $y$ del $5 \%$ en mujeres (13). La OMS afirma que el $80 \%$ de los casos de diabetes tipo 2 se puede prevenir mediante intervenciones sencillas y económicamente eficientes (12). Por lo cual es de vital importancia tener un mayor impacto en la aplicación de las guías y del seguimiento de los pacientes para obtener un mejor control de los pacientes pertenecientes a estos programas.

\section{Conclusiones}

En el programa de hipertensos con un cumplimiento de la guía del $67,8 \%$ se logra un control del $79 \%$ de los pacientes que se encuentran inscritos, cifra que está por encima del informe nacional de calidad de la atención en Salud del 2009 que mostro un control de 62-72\%, según informes de las instituciones prestadoras de salud. Pero aun así el cumplimiento de la guía es bajo, si se aumentara esto impactaría directamente en el control de los pacientes y en la disminución de la progresión de la enfermedad.

En el programa de pacientes diabéticos evaluados se encontró un cumplimiento de la guía del $62,8 \%$ y un control de la patología menor al 1\%, mientras que el $51 \%$ estaba en aceptable control, cuando se esperaba que el $20 \%$ de la población estuviera en control de acuerdo a lo reportado en los análisis de la Cuenta de Alto Costo. Es imperativo mejorar la aplicación de la guía en esta población, debido al bajo control que se encuentra en el programa, esto permite que se disminuyan los costos de manejo y la progresión de la patología en esta población.

La Asociación Latinoamericana de Diabetes (ALAD) plantea intervención en tres niveles para el manejo de la diabetes mellitus, prevención primaria en la que destacan la educación y manejo médico de los diversos factores contribuyentes al desarrollo de esta enfermedad. En cuanto a la prevención secundaria, se hace énfasis en prevenir las complicaciones y retardar la progresión de la enfermedad. La prevención terciaria la enfocan en evitar la discapacidad como por ejemplo la enfermedad renal crónica. Intervenciones que deben estar implementadas en los programas de promoción y prevención de la enfermedad para poder impactar de manera importante en el control de los pacientes con diabetes mellitus.
Uno de los determinantes del control de las enfermedades crónicas es el conocimiento de los pacientes de su enfermedad y la adherencia al tratamiento, por tal razón se debe informar a los pacientes en términos acordes a su nivel educativo sobre su enfermedad, la importancia de su seguimiento y control periódico. Además de buscar estrategias para evaluar este conocimiento en los pacientes y de la misma forma mejorarlo.

Se debe capacitar periódicamente al personal de la salud que forma parte del programa y realizar actualización de los temas relacionados con los programas de hipertensión y diabetes.

Es necesario hacer un comité de calidad para la revisión de historias clínicas periódicamente y realizar la correspondiente retroalimentación al personal a cargo del programa, para hacer más clara la información que se debe consignar, hacerla más legible y fácil de consultar, además de hacer sistematizada cada valoración médica al paciente, solicitando las ayudas diagnósticas e interconsultas de acuerdo a lo establecido en las guías de manejo.

Por último es necesario crear un conciencia política a nivel de las entidades rectoras y conductoras del Sistema, con el fin de implementar directrices enfocadas a la adecuada implementación de las guías por parte de los aseguradores en sus programas de crónicos, así como a la correcta aplicación de las guías en la población de usuarios por parte de las instituciones prestadoras de servicios de salud.

Conflicto de Interés: Los autores expresan que no presentan ningún conflicto de interés en la publicación de los resultados de esta investigación.

Financiación: Universidad Militar Nueva Granada, Proyecto MED 975.

\section{Referencias}

1. Organización Mundial de la Salud. 61 $1^{\mathrm{a}}$ Asamblea Mundial de la Salud, 18 de abril de 2008. Prevención y control de las enfermedades no transmisibles: aplicación de la estrategia mundial. Disponible en: http://apps.who.int/gb/ebwha/pdf_files/A61/A61_8-sp.pdf

2. Ministerio de la Protección Social (2010). Guía de Atención de la Hipertensión Arterial. Bogota. Disponible: http://www.nacer. udea.edu.co/pdf/libros/guiamps/guias13.pdf

3. Ministerio de la Protección Social (2010). Guía de Atención de la Diabetes Mellitus. Bogota. Disponible en: http://www.nacer.udea. edu.co/pdf/libros/guiamps/guias17.pdf

4. Situación de la enfermedad renal crónica en Colombia 2009. Cuenta de Alto Costo - Fondo Colombiano de Enfermedades de Alto Costo. 2010 
5. Ministerio de la Protección Social (2009) 1er. Informe Nacional de Calidad de la Atención en Salud. "INCAS Colombia 2009". Bogotá, D.C. Colombia. Disponible en: https://www.sos.com. co/ArchivosSubidos/Internet/Publicaciones/SAR/6\%20MPS\%20 INCAS\%20FINAL\%2024\%20junio\%20IMPRESION.pdf

6. Franklin S., Jacobs M., Wong N., L'Italien G., Lapuerta P. Predominance of isolated systolic hypertension among middle-aged and elderly US hypertensives: analysis based on National Health and Nutrition Examination Survey (NHANES) III. Hypertension 2001 Mar;37(3):869-74.

7. Nuria V.,Ferrera, Dante H. Moine, Yañez C. Hipertensión arterial: implementación de un programa de intervención de control y adherencia al tratamiento en un Centro de Atención Primaria de la Salud de la ciudad de Paraná. Rev.Fed .Arg. Cardiol 2010; 39 (3): 194-203

8. Ariza A., Castaño J. et al. Evaluación de la historia clínica de pacientes hipertensos de acuerdo a indicadores de calidad del programa de hipertensión arterial, Clínica Assbasalud La Enea (ESE) 2004-2007. Vol. 9 N 1, 2009.

9. Prado H. Programa educativo sobre hipertensión arterial a los pacientes que acuden al club de hipertensos la sabanita. Ciudad Bolívar- Venezuela. 2010

10. Papazafiropoulou A., Skliros E., Sotiropoulos A., Papafragos C., Gikas A. Prevalence of target organ damage in y pertensive subjects attending primary care: C.V.P.C. study (epidemiological cardio-vascular study in primary care). BMC Family Practice 2011, Disponible en: http://www.biomedcentral.com/14712296/12/75

11. López L., Morales A., Ortiz M., Portilla M. Estudio comparativo de daño a órgano blanco entre hipertensos controlados y no contro- lados, comprendido entre las edades de 35 - 60 años que consultaron al hospital de caldas entre los años 1998 - 2002. Disponible en: http://www.umanizales.edu.co/publicaciones/campos/medicina/archivos_medicina/html/publicaciones/edicion_8/4_comparativo_organ.pdf

12. Federación internacional de diabetes. PLAN MUNDIAL CONTRA LA DIABETES 2011-2021.

13. Sánchez P. Control Metabólico en Pacientes Diabéticos Tipo 2: grado de Control y nivel de Conocimientos (Estudio Azuer). REV CLÍN MED FAM 2011; 4 (1): 32-41

14. Tesfaye S., Chaturvedi N., Eaton S., Ward J., Manes C., Tirgoviste C, Witte D., Fuller J., Vascular Risk Factors and Diabetic Neuropathy N Engl J Med 2005;352:341-50. Disponible en: http://www. nejm.org/doi/pdf/10.1056/NEJMoa032782

15. Gotto, J., Bailey K., D. Gohdes, Haffner S., Hiss R., JamersonK., LeeK., NathanD., SowersJ., WaltersL.. Effects of Medical Therapies on Retinopathy Progression in Type 2 Diabetes. nengl j med 363;3 nejm.org july 15, 2010. Disponible en: http://www.nejm. org/doi/pdf/10.1056/NEJMoa1001288

16. Sánchez D., Cuéllar P., Delgadillo M., Durán E., Deveze M. Prevalencia de daño renal en pacientes diabéticos y/o hipertensos mediante prueba tamiz (RAC) en una clínica de Guanajuato. Rev Latinoamer Patol Clin, Vol. 59, Núm. 1, pp 28-34 - Enero - Marzo, 2012. Disponible en: http://www.medigraphic.com/pdfs/patol/ pt-2012/pt121f.pdf

17. Ministerio de la Proteccion Social. Criterios para identificar patologías de alto costo en Colombia. Documento de trabajo Cuenta de Alto Costo 2011. Disponible en-. http://www.minsalud.gov.co/ sites/rid/Lists/BibliotecaDigital/RIDE/INEC/CAC/ALTO_COSTO_FINAL_070911.pdf 